\title{
AAFP Convocation of Practices and Network: Report from the 2005 Annual Meeting
}

\author{
Kevin Peterson, MD, MPH, Elizabeth W. Staton, MSTC, and Walter L. Calmbach, MD
}

The sixth annual AAFP Convocation of Practices and Network, a joint meeting of the AAFP National Research Network (AAPFNRN) and the Federation of Practice-Based Research Networks (FPBRN), was held March 3 to 6, 2005, at the Cheyenne Mountain Conference Resort in Colorado Springs, CO. Conference attendees, many of whom are practicing clinicians who participate in practice-based research, heard talks related to the Future of Family Medicine-the theme for the Convocation.

Holding the Convocation in Colorado Springs represented a return of sorts for AAFPNRN members who were active in the Ambulatory Sentinel Practice Network. ASPN, one of the earliest practice-based research networks in the United States, held its annual meeting at Cheyenne Mountain for many years. Similar to APSN, the AAFPNRN has a broad reach-including 300 physicians in 180 practices across 45 US states and 4 Canadian provinces and caring for an estimated 750,000 patients.

At this year's Convocation, Dr. Michael Fleming, Chair of the AAFP Board of Directors, presented his vision of the "Family Medicine Office of the Future." Several experts spoke about incorporating information technology into PBRN research, as well as how the Future of Family Medi-

Submitted, 22 April 2005.

From the Steering Committee, Federation of PracticeBased Research Networks, and Department of Family Medicine and Community Health, University of Minnesota, Minneapolis (KP), AAFP National Research Network and the Department of Family Medicine, University of Colorado at Denver and Health Sciences Center, Denver (EWS), and the Department of Family \& Community Medicine, South Texas Ambulatory Research Network (STARNet), University of Texas Health Science Center at San Antonio (WLC).

Conflict of interest: KP is the president and WLC is the president-elect of the Federation of Practice-Based Research Networks [both unpaid, volunteer positions]. EWS is a paid employee of the AAFP National Research Network.

Corresponding author: Kevin Peterson MD, MPH, Department of Family Medicine and Community Health, Suite 220, 925 Delaware Street SE, Minneapolis, MN 55414 (email: peter223@umn.edu). cine report can drive the research agenda of practice-based research.

One of the prominent themes discussed during the Convocation's plenary and breakout sessions was the significance of patient-centered care and how it fits in the New Model of Family Medicine. In contrast to a more traditional perspective, patient-centered care puts the patient, not the physician, at center stage. Physicians using this approach will tailor care so that it is appropriate (eg, culturally, linguistically, etc) for each patient. To provide patient-centered care, physicians explore both the disease and the illness experience, understanding the whole person. They must seek common ground, incorporate prevention and health promotion, enhance the patient-physician relationship, and be realistic with all patients. The ultimate goal is to enhance the medical encounter so that goals and needs of both patients and physicians are successfully addressed.

One of the most popular offerings at the Convocation was the breakout sessions, which fostered small group interaction, dialogue, and collaboration. Breakout sessions included discussion of potential cooperation between AAFPNRN and regional networks, presentation of preliminary and final AAFPNRN study results, and exploration of potential study topics.

At the Patient Safety discussion group, family physicians and study coordinators who participated in previous AAFPNRN patient safety studies discussed safety in primary care. Most of the practices represented by this focused discussion have made concerted efforts to improve patient safety over the past year. Some of these endeavors include using the AAFP patient safety taxonomy for morbidity and mortality conferences; establishing tracking systems for laboratory tests, radiographs, and other tests; developing a new protocol for handling emergencies; establishing a flow sheet to handle duplicate prothrombin tests; and developing a system for 
following abnormal Papanicolaou smears through to treatment. Several of these activities were developed as a result of the practices' participation in one of the AAFPNRN patient safety studies. This group stated unanimously that the AAFPNRN should continue research in patient safety, perhaps through an error-reporting web site with practicespecific feedback.

The Federation of Practice Based Research Networks (FPBRN) jointly sponsors the Convocation of Practices with the National Research Network. Formed in 1997 from a loosely affiliated group of ASPN-associated networks, the group has grown from 12 networks to 53 primary care practice-based research networks (PBRN) with over 6500 members and a potential patient population of over 16 million. Although more than $65 \%$ of Federation members are family physicians, the FPBRN includes members from all areas of primary care, including members from the American College of Physicians, the American Academy of Pediatrics, nurse practitioners, physician assistants, and primary care researchers. The mission of the FPBRN is to advocate for practice-based research, build capacity for practice-based research, and to foster collaboration and communication among the member networks. For several years the Federation has helped organize 2 tracks held in conjunction with the AAFP National Research Network Convocation of Practices: (1) PBRN methodology and (2) presentations of completed research projects or research-in-progress. Some highlights of the 2005 "Federation Track" include:

Kevin Peterson (University of Minnesota) presented a session describing implementation of the electronic Primary Care Research Network (ePCRN) Initiative. This initiative is funded by a National Institutes of Health Roadmap grant and is designed to create and test an electronic network administered by the FPBRN that supports both research and clinical medicine in primary care practices. The purpose of this session was 2-fold: to discuss the general features and goals of the project and to conduct a demonstration and instruction on the use of the site for the network directors and study coordinators of the participating networks. Ten member networks are currently participating. The project is planned in 3 stages: (1) create an infrastructure to support randomized clinical trials among member networks, (2) create an infrastructure to support translation of research into practice, and (3) establish high-speed Internet-2 connectivity to facilitate training and multisite PBRN studies.

Jack Westfall (University of Colorado Health Science Center) presented a workshop on the design and implementation of the High Plains Research Network Community Advisory Council. The purpose of the workshop was to provide concrete steps for other practice-based research networks considering participatory research. Dr. Westfall reviewed the creation of the Community Advisory Council, how it evolved, and how it dealt with the problems and challenges inherent in community participatory research across a wide geographic area. Keys to success included clear expectations and mutual respect among council members, logistic support, and listening skills. A highlight of the session was the presence and input of local members of the Community Advisory Council during the workshop.

Margaret Handley (University of California San Francisco) presented a seminar on methods used in the IDEALL study (Improving Diabetes Effects Across Language and Literacy). The purpose of the session was to describe the development of an integrated database that has been the backbone of the IDEALL Study. IDEALL is an ongoing randomized clinical trial examining 2 forms of diabetes self-management support (group medical visits versus an automated telephone system that contacts patients) and usual care, in 3 languages. As described, the IDEALL study is a good example of RE-AIM technique. RE-AIM is a method of evaluating the potential impact of an intervention on the community described by Russell Glasgow. The elements of the evaluation are: Reach into the target population, Efficacy or effectiveness, Adoption by target settings or institutions, Implementation-consistency of delivery of intervention, and the Maintenance of intervention effects in individual patients and populations over time. IDEALL extended its reach by the purposeful selection of 4 clinics and 3 languages, testing 2 interventions (automated phone management or group clinics with a physician, diabetes educator, and pharmacist) versus usual care.

Jim Mold (University of Oklahoma Health Science Center) and Chris Van Weel (Nijmegen University Academic Network, Family Medicine) presented a seminar on the conduct of longitudinal 
cohort studies in PBRNs. This session described both concurrent cohort studies (ie, time-forward design) and nonconcurrent cohort studies (ie, chart review, identify date of diagnosis, and follow cases forward). Concurrent cohort studies are an important method in PBRN research because of our interest in continuity and its effect on the content and process of care. Nonconcurrent cohort studies are an attractive method for PBRN research because of the rich data available in well-kept records, but these are limited by the retrospective nature of their design. Drs. Mold and Van Weel presented examples of both types of cohort studies and led a discussion of the positive and negative aspects of both.

Chet Fox (SUNY at Buffalo) led a panel composed of James Mold, Myra Crawford (University of Alabama), and Bennett Parnes (University of Colorado Health Sciences Center) to discuss the role and importance of the Practice Enhancement Assistant (PEA). Key qualities of the successful PEA include honesty, integrity, adaptability, perseverance, patience, and intelligence. For the participating practice, the PEA is "an agent of change, a coordinator, a cross-pollinator of good ideas, a resource-provider, an information giver, researcher, adviser, and mentor." Among the many benefits of PEAs, they know the practice staff, are familiar with the practice structure and physical space, and are well-positioned to provide feedback to member practices and their staff.

Additional sessions on the "Federation track" were presented by Alan Adelman (Penn State University), Debbie Allen (Indiana University), Margaret Love (University of Kentucky), John Ryan (University of Miami), Jonathon Temte (University of Wisconsin), and Jim Werner (Case Western Reserve University). 